\title{
The long non-coding RNA uc.4 influences cell differentiation through the TGF-beta signaling pathway
}

\author{
Zijie Cheng ${ }^{1,5}$, Qijun Zhang ${ }^{2,5}$, Anwen Yin ${ }^{1,5}$, Mengwen Feng ${ }^{1}$, Hua $\mathrm{Li}^{1}$, Hailang $\mathrm{Liu}^{3}$, Yun $\mathrm{Li}^{4}$ and \\ Lingmei Qian ${ }^{1}$
}

In a previous study, we screened thousands of long non-coding RNAs (IncRNAs) to assess their potential relationship with congenital heart disease (CHD). In this study, uc.4 attracted our attention because of its high level of evolutionary conservation and its antisense orientation to the CASZ1 gene, which is vital for heart development. We explored the function of uc.4 in cells and in zebrafish, and describe a potential mechanism of action. P19 cells were used to investigate the function of uc.4. We studied the effect of uc. 4 overexpression on heart development in zebrafish. The overexpression of uc. 4 influenced cell differentiation by inhibiting the TGF-beta signaling pathway and suppressed heart development in zebrafish, resulting in cardiac malformation. Taken together, our findings show that uc. 4 is involved in heart development, thus providing a potential therapeutic target for CHD.

Experimental \& Molecular Medicine (2018) 50, e447; doi:10.1038/emm.2017.278; published online 16 February 2018

\section{INTRODUCTION}

Congenital heart disease (CHD) is the most common birth defect, with an estimated incidence of $2-3 \% .^{1,2} \mathrm{CHD}$ can lead to both fetal growth retardation as well as spontaneous abortion and stillbirth. CHD can seriously affect the quality of life of children and puts heavy economic and social burdens on the families and society. ${ }^{3}$ Despite the remarkable progress in scientific research and clinical treatment, the mechanism of CHD remains poorly understood.

In recent years, new evidence has shown that $\sim 95 \%$ of human genome transcripts are non-coding RNAs. ${ }^{4}$ Long noncoding RNAs (lncRNAs) are a subtype of non-coding RNAs (ncRNAs) with transcripts longer than 200 nucleotides. LncRNAs are involved in several biological and pathological processes, including vascularization, ${ }^{5}$ osteoarthritis, ${ }^{6}$ diabetes ${ }^{7}$ and tumorigenesis. ${ }^{8}$ Ultraconserved elements are specific lncRNAs that are completely conserved among the human, rat and mouse genomes. ${ }^{9}$ Recent studies have delineated the essential role of ultraconserved elements (UCEs) that regulate adipogenesis ${ }^{10}$ and tumor formation. ${ }^{11,12}$ All of these lines of evidence emphasizes the relevance of UCEs in disease etiology.
Heart development involves formation of the early primitive heart tube, cardiomyocyte differentiation and heart morphogenesis. The role and mechanism of lncRNA in cardiac development have been primarily described in three reports on Braveheart, ${ }^{13}$ and Fendrr, ${ }^{14}$ which are involved in mouse cardiac development, and Terminator, ${ }^{15}$ a vertebrate lncRNA that is important for cardiovascular development. There is no doubt that exploring the role and mechanism of lncRNA in cardiac development will deepen our understanding of CHD. ${ }^{16}$ However, the relationship between UCEs and CHD remains unknown.

In our previous study, we screened thousands of $\operatorname{lncRNAs}$ to investigate their relationship with $\mathrm{CHD} .{ }^{17}$ Among those IncRNAs, uc.4 attracted our attention due to its high level of conservation and its antisense orientation to the CASZ1 gene, which is essential for cardiac morphogenesis and development. In the present study, we performed bioinformatics analyses and assessed the effect of uc.4 on the differentiation of P19 cells as well as the potential relationship between uc. 4 and CASZ1. We also assessed the effects of uc.4 on heart development in zebrafish. We showed that uc.4 exerts its activity through the TGF-beta signaling pathway. These data suggested that uc.4

\footnotetext{
${ }^{1}$ Department of Cardiology, The First Affiliated Hospital of Nanjing Medical University, Nanjing, China; ${ }^{2}$ Department of Cardiology, YinZhou Hospital Affiliated to Medical School of Ningbo University, Ningbo, China; ${ }^{3}$ Huai An First People's Hospital, HuaiAn, China and ${ }^{4}$ Department of Pharmacy, Obstetrics and Gynecology Hospital Affiliated to Nanjing Medical University, Nanjing, China

${ }^{5}$ These authors contributed equally to this work.

Correspondence: Dr Y Li, Department of Pharmacy, Obstetrics and Gynecology Hospital Affiliated to Nanjing Medical University, Nanjing 210029, China. E-mail: 407457529@qq.com

or Professor L Qian, Department of Cardiology, The First Affiliated Hospital of Nanjing Medical University, Nanjing 210029, China.

E-mail: Imqian@njmu.edu.cn
}

Received 3 May 2017; revised 26 August 2017; accepted 31 August 2017 
may play an important role in heart development and provide a possible mechanism of $\mathrm{CHD}$.

\section{MATERIALS AND METHODS}

\section{Cell culture and differentiation}

Murine embryonal carcinoma cells (P19) were obtained from American Type Culture Collection (Manassas, VA, USA) and cultured in Alpha Modification of Eagle's medium (Gibco, Grand Island, NY, USA) containing 10\% fetal bovine serum (Gibco), $100 \mathrm{U} / \mathrm{ml}$ penicillin and $100 \mu \mathrm{g} \mathrm{ml}^{-1}$ streptomycin in $5 \% \mathrm{CO}_{2}$ at $37^{\circ} \mathrm{C}$. Following $1 \%$ DMSO induction, the cells were aggregated at days $3-4$. After 7 days of aggregation in bacteriological dishes, the cell clusters were transferred to six-well culture plates in Alpha Modification of Eagle's medium containing $10 \%$ fetal bovine serum. The medium was replaced every 2 days. The cells were harvested on differentiation days $0,4,8$ and 10 . We observed and photographed the morphological changes in the P19 cells using an inverted microscope (Nikon, Tokyo, Japan).

\section{Rapid amplification of cDNA ends}

$5^{\prime}$ and $3^{\prime}$ rapid amplification of CDNA ends (RACE) was performed on RNA isolated from mouse heart using a SMARTer RACE $5^{\prime} / 3^{\prime}$ Kit (Clontech, Mountain View, CA, USA), following the manufacturer's instructions. The RACE PCR products were separated on a $1 \%$ agarose gel and cloned into the pUC57 vector. The transcription start and end sites of uc. 4 were mapped by sequencing.

\section{Nuclear and chromatin RNA fraction}

Nuclear and cytoplasmic fractions of P19 cells were partitioned using a PARIS Kit (Thermo Fisher Scientific, Carlsbad, CA, USA). Overall, $10^{7}$ cultured cells were collected, placed on ice and resuspended with $500 \mu \mathrm{l}$ of ice-cold cell fractionation buffer. The cells were then gently resuspended by vortexing or pipetting, and were incubated on ice for another $10 \mathrm{~min}$. The samples were centrifuged at $500 \mathrm{~g}$ for $5 \mathrm{~min}$, and the cytoplasmic fraction was then carefully aspirated away from the nuclear pellet fraction. Washing the pellet twice in ice-cold cell fractionation buffer prevented contamination of the nuclear fraction with the cytoplasmic fraction.

Lentivirus production and establishment of a stable cell line The uc.4 lentiviral overexpression vector was constructed by GenePharma (Shanghai, China). Lentiviruses were amplified in 293T cells and concentrated using polyethylene glycol (System Biosciences, LLC, Palo Alto, CA, USA). For the overexpression experiments, the infection efficiency was verified in infected P19 cells by quantitative real-time polymerase chain reaction (qRT-PCR). Puromycin (Sigma, Shanghai, China) selection was used to establish stable cell lines.

\section{Cell cycle and apoptosis analysis of P19 cells}

P19 cell proliferation was measured using a CCK8 assay kit (Dojindo, Japan).The cells were seeded (500 cells per well) into 96-well plates, and CCK8 was added $0,24,48,72$ and $96 \mathrm{~h}$ later for $2 \mathrm{~h}$, after which the OD values were recorded. P19 cells deprived of fetal bovine serum were starved for $12 \mathrm{~h}$ before recovery in complete medium for another 24 and $48 \mathrm{~h}$. The cells were either fixed in 50\% ethanol for cell cycle analysis or harvested with EDTA-free trypsin for apoptosis analysis. The cells were resuspended in $500 \mu \mathrm{l}$ of binding buffer and stained with $10 \mu \mathrm{l}$ of annexin V-APC/7-ADD at room temperature for $10 \mathrm{~min}$. These samples were analyzed using flow cytometry using Kaluza Software (Beckman Coulter, Indianapolis, IN, USA). The data acquisition (10000 events collected per sample) was performed following the manufacturer's instructions.

\section{Gene analysis and western blotting}

Total RNA was extracted from either cultured cells or frozen tissues using TRIzol reagent (Thermo Fisher Scientific). The concentration of RNA was determined by measuring the absorbance ratio of 260$1280 \mathrm{~nm}$ using a NanoDrop ND-1000 spectrophotometer (Thermo Fisher Scientific), and the integrity of the RNA was checked using gel electrophoresis. The reverse transcription of RNA was performed using a PrimeScript RT reagent Kit with gDNA eraser (RR047A; Takara, Tokyo, Japan), and cDNA was analyzed by qRT-PCR using SYBR Premix Ex Taq (RR420A; Takara). The data were normalized to the levels of GAPDH and further analyzed using the $2^{-\Delta \Delta C T}$ method. The sequences for all of the primers used for $\mathrm{qPCR}$ are listed in Supplementary Table 1. For the western blot analysis, protein was solubilized from cell lysates at four different time points using RIPA buffer and quantified using a BCA Protein Assay Kit (23229; Thermo Fisher Scientific). A total of $40 \mu \mathrm{g}$ protein was separated on a $10 \%$ gel using SDS-PAGE and transferred to nitrocellulose membranes (Millipore, Billerica, MA, USA). Immunopositive bands were detected using a FluorChem M System (ProteinSimple, San Jose, CA, USA). Antibodies targeting cardiac troponin $\mathrm{T}$ (cTnT, ab92546), myocyte enhancer factor 2C (MEF2C, ab197070), GATA-binding protein 4 (GATA4, ab84593), phosphorylation of mothers against decapentaplegic homolog 2 (smad2-P, ab188334), phosphorylation of mothers against decapentaplegic homolog 3 (smad3-P, ab52903), total mothers against decapentaplegic homolog 2 (smad2-T, ab33875) and total mothers against decapentaplegic homolog 3 (smad3-T, ab40854) were purchased from Abcam, Shanghai, China.

\section{Zebrafish maintenance}

The Tübingen zebrafish strain used in this study was obtained from the Model Animal Research Center of Nanjing University (Nanjing, Jiangsu, China). Zebrafish were raised at $28^{\circ} \mathrm{C}$ in a rotating system that continuously percolates, UV treats and aerates the circulating water. The embryos were acquired from the natural spawning of wildtype (WT) adults and were raised at $28 \pm 1^{\circ} \mathrm{C}$. Morphological features were used to determine the embryonic developmental stage. ${ }^{18}$ Embryos older than $24 \mathrm{~h}$ post fertilization (hpf) were incubated in $0.003 \%$ phenylthiourea to inhibit pigment formation.

\section{Expression vectors and microinjection}

The uc.4 overexpression and standard negative control (NC) vectors were purchased from GenePharma (Shanghai, China) and dissolved in RNase-free water at $500 \mathrm{ng} / \mu \mathrm{l}$. The injection of 100,200 or $400 \mathrm{pg}$ of uc.4 mRNA into single- to four-cell-stage zebrafish embryos was performed using back-filled fine borosilicate glass capillary needles. Following microinjection, the embryos were incubated at $28 \pm 1{ }^{\circ} \mathrm{C}$, and the embryo medium was replaced. Degenerating embryos were removed daily.

\section{In situ hybridization}

A digoxigenin-labeled oligonucleotide probe with the following sequence was generated for in situ hybridization: $5^{\prime}$ TATGCTGTGTGGGCTAAGATGGCA-3'. All of the embryos were fixed in $4 \%$ paraformaldehyde at $4{ }^{\circ} \mathrm{C}$ overnight and then dehydrated through graded methanol solutions, permeabilized by proteinase $\mathrm{K}$, prehybridized, and hybridized overnight at $65^{\circ} \mathrm{C}$ in $50 \%$ formamide buffer with DIG-labeled probes. After being washed, the samples were 

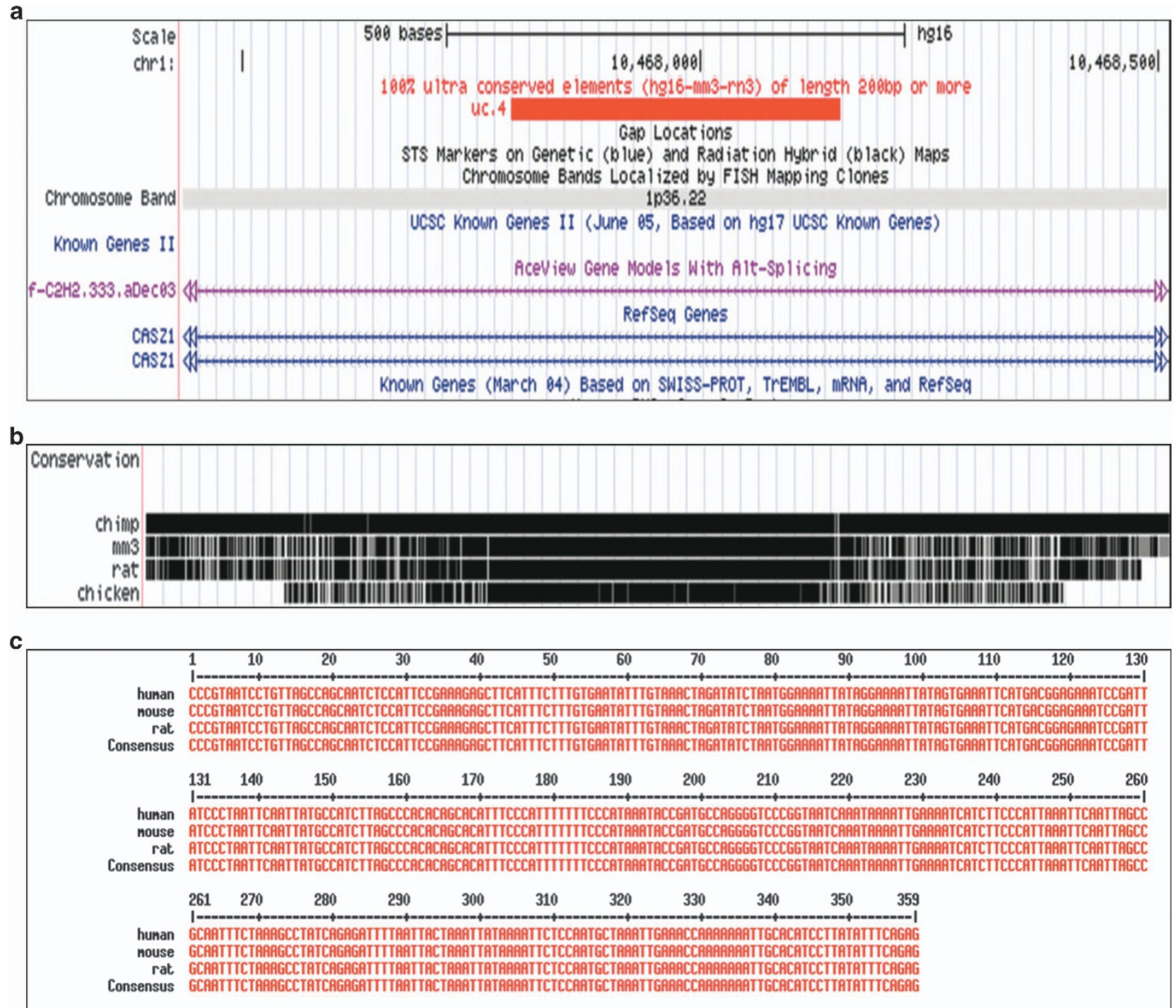

d

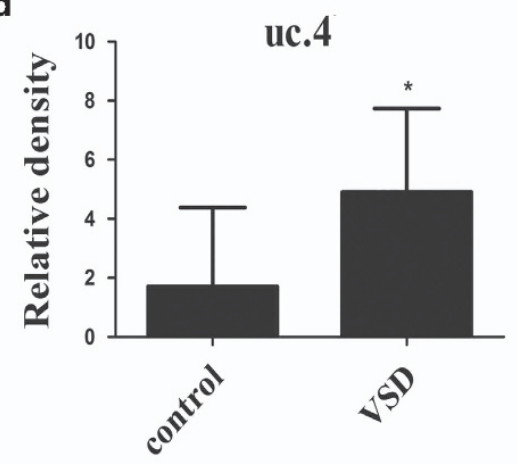

e

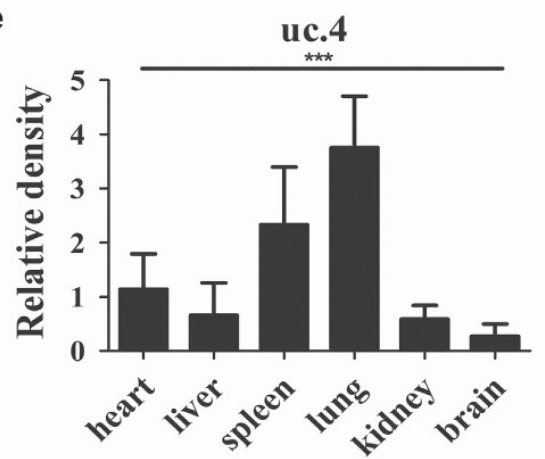

f

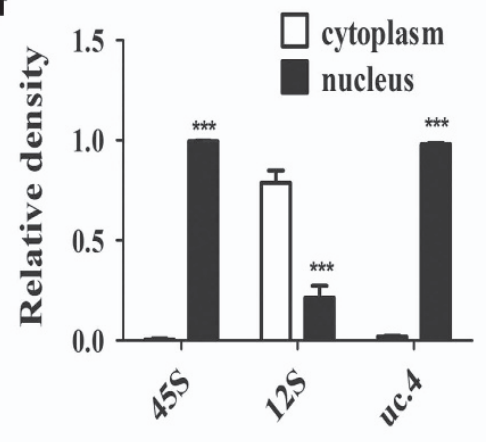

Figure 1 The genomic location and bioinformatics analyses of uc.4. (a) Bioinformatics analyses of uc. 4 using the UCSC genome browser (http://genome.ucsc.edu). (b) Conservation of uc.4 between chimp, mm3, rat and chicken. Chimp, chimpanzee; mm3, mouse. (c) The results sequencing that was performed to verify the conservation of uc.4. (d) uc.4 expression levels were significantly increased in human fetal VSD heart tissues. ${ }^{*} P<0.05$ versus controls, Student's $t$-test. (e) The profile of uc.4 expression in six organs (heart, liver, spleen, lung, kidney and brain). The expression level of uc.4 was higher in lung tissue than other tissues. (f) qRT-PCR analyses of uc.4 in cytoplasmic and nuclear fractions of P19 cells. 45S rRNA and mitochondrial 12S rRNA served as positive controls. $* * * P<0.001$, Student's $t$-test. There were three replicates for each experiment. Data are presented as the means \pm s.d. qRT-PCR, quantitative real-time polymerase chain reaction; VSD, ventricular septal defect. 
incubated with phosphatase-conjugated antibody against digoxigenin at $4{ }^{\circ} \mathrm{C}$ overnight. The NC and uc.4-overexpressing embryos were processed in parallel.

\section{Observation of malformations and heart rate}

Embryos at different time points (48, 72 and $96 \mathrm{hpf}$ ) were photographed with an Olympus DP71 digital camera (Olympus, Tokyo, Japan) to observe heart development. The images were assembled using Adobe Photoshop CS6 software (AdobeSystems, San Jose, CA, USA).The larvae were anesthetized in tricaine to estimate the heart rate. The heart rate was calculated by counting the number of continuous contractions, beginning and ending at the end diastole.

\section{Microarray \\ Total RNA from differentiated d10 cells was extracted using TRIzol Reagent (Life Technologies, Carlsbad, CA, US), following the manu- facturer's instructions. The RIN (RNA integrity number) was deter- mined to assess RNA integrity using an Agilent Bioanalyzer 2100 (Agilent Technologies, Santa Clara, CA, USA). Qualified total RNA was further purified using an RNeasy Mini Kit (QIAGEN, GmBH, Germany) and an RNase-Free DNase Set (QIAGEN). Total RNA was amplified and labeled with a Low-Input Quick Amp Labeling Kit, One-Color (Agilent Technologies). Labeled cRNA was purified using an RNeasy mini kit (QIAGEN). The slides were scanned on an Agilent Microarray Scanner (Agilent Technologies) using the default settings (Dye channel: Green, scan resolution $=3 \mu \mathrm{m}$, photomultiplier tube (PMT) 100\%, 20 bit). The data were extracted using the Feature Extraction software 10.7 (Agilent Technologies). The raw data were normalized using the Quantile algorithm, limma package in R.}

\section{Statistical analysis \\ The quantitative data are expressed as the means \pm s.d. The values were compared using Student's $t$-test. All of the statistical analyses were performed using SPSS 13.0 software (Chicago, IL, USA). A P- value of $<0.05$ was considered statistically significant.}

\section{RESULTS}

\section{Bioinformatics analysis of uc. 4}

Ultraconserved elements in the human genome are defined as a series of at least $200 \mathrm{bp}$ of DNA that are identical with the corresponding regions in the mouse and rat genomes. uc. 4 is an intronic lncRNA located on chromosome $1 \mathrm{p}$ in the human genome and on chromosome $4 \mathrm{p}$ in the mouse genome (Figure 1a). Multiple alignment analysis of the uc.4 locus revealed that this lncRNA is highly conserved in mouse, rat, human and chicken (Figure 1b) based on the University of California, Santa Cruz (UCSC) genome browser (Santa Cruz, CA, USA; http://genome.ucsc.edu). The sequence of the uc.4 locus showed $100 \%$ identity among human, mouse and rat (Figure 1c). We used Coding Potential Calculator analyses (Peking University, Beijing, China) to distinguish non-coding RNAs from protein-coding RNAs based on the sequence features. uc. 4 had no credible protein-coding open reading frames, with a coding potential score of -1.30047 (Supplementary Figure 1A). These data suggest that uc.4 does not encode a peptide.

\section{Characterizing the ultraconserved lncRNA uc. 4}

RACE was performed to characterize the $5^{\prime}$ and $3^{\prime}$ ends of this transcript. However, we failed to obtain a full-length sequence through RACE (Supplementary Figure 1B), suggesting that the length of uc. 4 transcript is $359 \mathrm{nt}(9)$. qRT-PCR revealed that uc.4 expression was significantly higher in human fetal ventricular septal defect tissues than in normal fetal tissues (Figure 1d). Furthermore, transcripts of uc.4 could be detected in multiple tissues in adult mice (Figure 1e). Assessment of the distribution indicated that uc.4 is primarily expressed in lung tissues, suggesting that this lncRNA could function not only in heart development but also in other tissues or cell lines. Further fractionation of P19 cells demonstrated that uc.4 was predominantly localized to the nucleus (Figure 1f). As a control, the localization of the $45 \mathrm{~S}$ rRNA precursor was determined, with the results showing that it primarily localized to the nuclear fraction. In contrast, the mitochondrial $12 \mathrm{~S}$ rRNA gene was primarily found in the cytoplasmic compartment.

\section{The effects of uc. 4 overexpression on cell proliferation cycle and apoptosis}

To explore the effects of uc. 4 overexpression, a uc. 4 lentiviral overexpression vector was constructed and used transfect P19 cells. The transfection efficiency was detected using fluorescence microscopy (Figure 2a). The expression of uc.4 was increased $\sim 70$-fold based on quantitative analysis (Figure $2 b$ ). The CCK- 8 assay was used to assess the proliferation of uc.4overexpressing P19 cells over a $96-\mathrm{h}$ period (Figure 2c). The flow cytometry analysis revealed no difference in the cell cycle of uc.4-overexpressing cells (Figure 2d). Annexin V-APC/7ADD staining and flow cytometry were used to assess apoptosis. Notably, uc.4 overexpression in P19 cells did not affect cell proliferation, the percentage of apoptotic cells or cell cycle kinetics compared with control cells (Figure 2e). Thus, uc. 4 is not required to maintain P19 cell self-renewal.

The overexpression of uc. 4 influenced P19 cell differentiation We examined whether uc.4 overexpression could influence P19 cell differentiation. Embryoid bodies were generated using 4 days of 1\% DMSO induction. Autonomous pulsation was observed on days 9-10 and can continue for 3-4 days. We detected the mRNA expression of cardiac markers, such as GATA4, cTnT, $\alpha$-MHC and MEF2C in these cells. Compared with the control group at the same time points, GATA4, cTnT, $\alpha-\mathrm{MHC}$ and MEF2C expression levels in the uc.4-overexpression group were downregulated during P19 differentiation into cardiomyocytes on days 8 and 10 (Figure 3a). Abnormal morphology of the embryoid bodies was visible in the uc. 4 overexpression group (Figure $3 b$ ). Variations in the size, shape and number of beats in the uc.4 overexpression group remained minimal during differentiation. Western blotting yielded similar results (Figures $3 c$ and $d$ ), verifying the decrease in the expression of cardiac-specific markers on days 8 and 10. These results revealed that the overexpression of uc. 4 could suppress the differentiation of P19 cells to myocardial cells. 


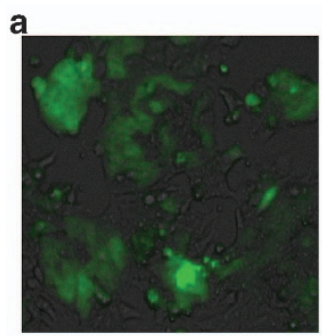

NC

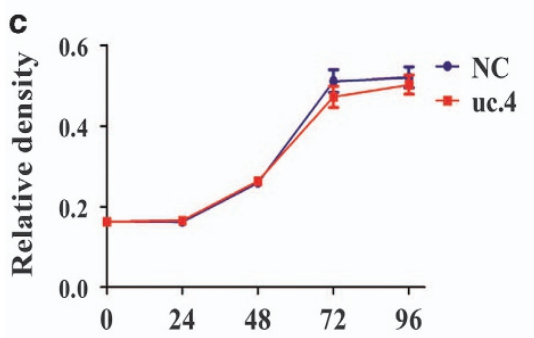

b

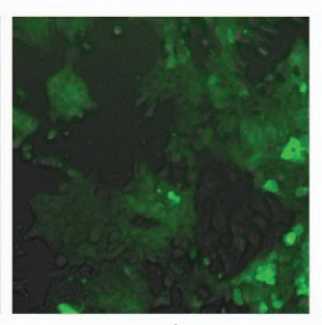

uc.4

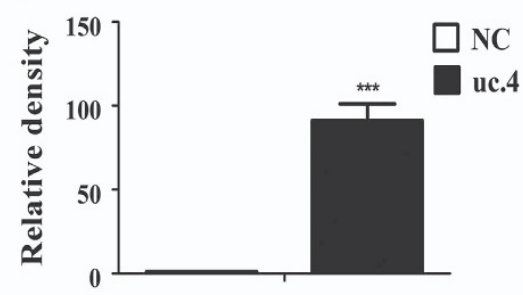

d

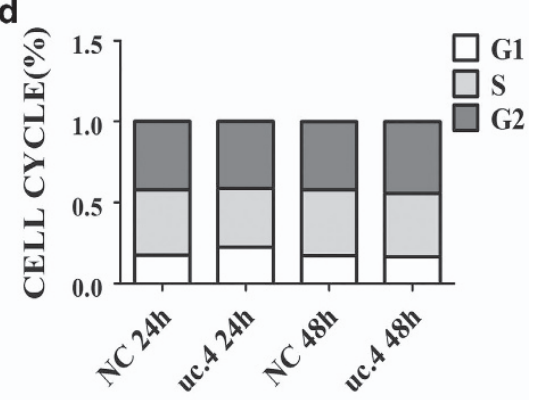

e
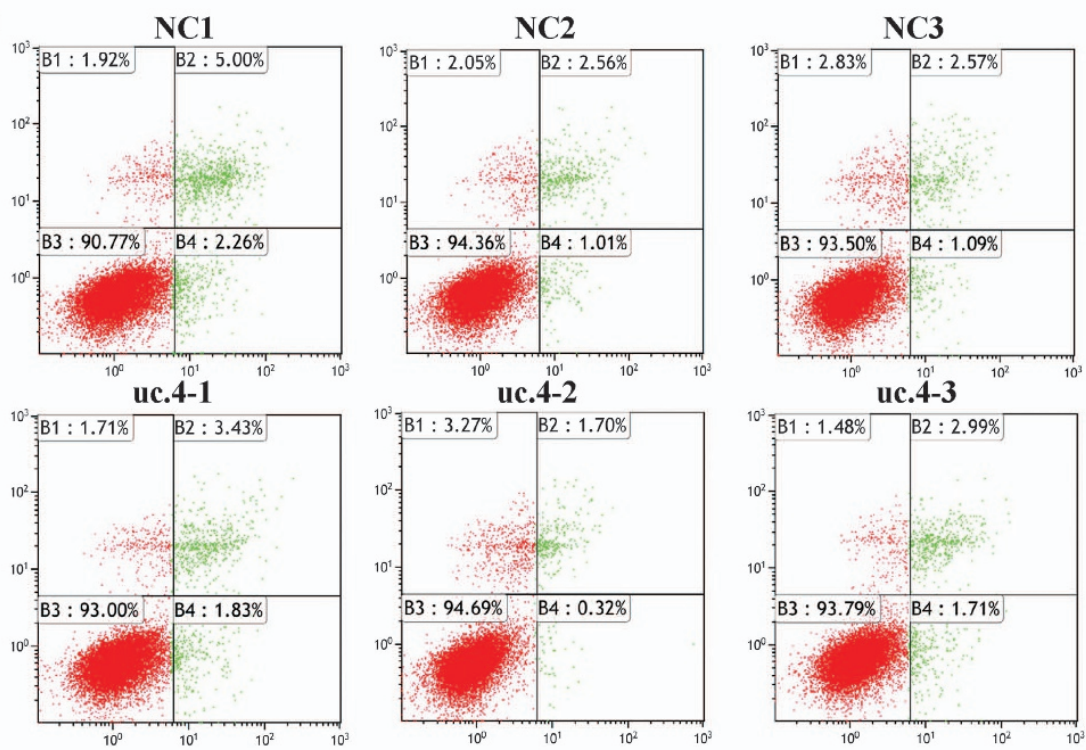

Figure 2 Functional analyses of uc.4. (a) Optical and fluorescence microscopy $(\times 100)$ were used to determine the lentivirus transfection efficiency of the uc.4 overexpression and control vectors via GFP expression cells after 2 weeks of puromycin selection. (b) uc.4 expression levels in P19 cells were significantly higher than in the NC groups. ${ }^{* *} P<0.001$, Student's $t$-test. (c) Proliferation was analyzed using CCK-8 at 0, 24, 48, 72 and $96 \mathrm{~h}$. The OD450 value represents the proliferation capacity. (d) The cell cycle was analyzed using flow cytometry. (e) Flow cytometry was performed to analyze apoptosis. Data are presented as the means \pm s.d. of three independent experiments. GFP, green fluorescent protein; NC, negative control.

\section{Lethality and malformation rates of uc.4-injected zebrafish} embryos

To observe the effect of uc.4 on zebrafish heart development, we injected different amounts (100, 200 and $400 \mathrm{pg})$ of uc. 4 mRNA into single cells in the embryonic phase. The death rates were observed at five different development stages $(6,24,48,72$ and $96 \mathrm{hpf}$. The results showed that the mortality of zebrafish embryos and larvae gradually increased with increasing mRNA amounts, indicating specific dose-dependent effects (Figure 4a). These findings revealed that the overexpression of uc. 4 can result in the aberrant development of zebrafish embryos (Figure 4b). On the basis of our evaluation of the morphological statistics, we chose a dose of $200 \mathrm{pg}$, for which the overexpression effect was verified by qRT-PCR. Specifically, the expression of uc. 4 was increased $\sim 40$-fold using quantitative analysis (Figure 4c). In situ hybridization experiments revealed that uc. 4 was primarily distributed in the atria of zebrafish embryos (Figure 4d).

\section{Effect of uc.4 overexpression on heart morphology and cardiac marker expression}

An overexpression vector and $\mathrm{NC}$ vector were injected into the embryo at the single-cell stage to observe the development of zebrafish over four different periods. The results showed that 

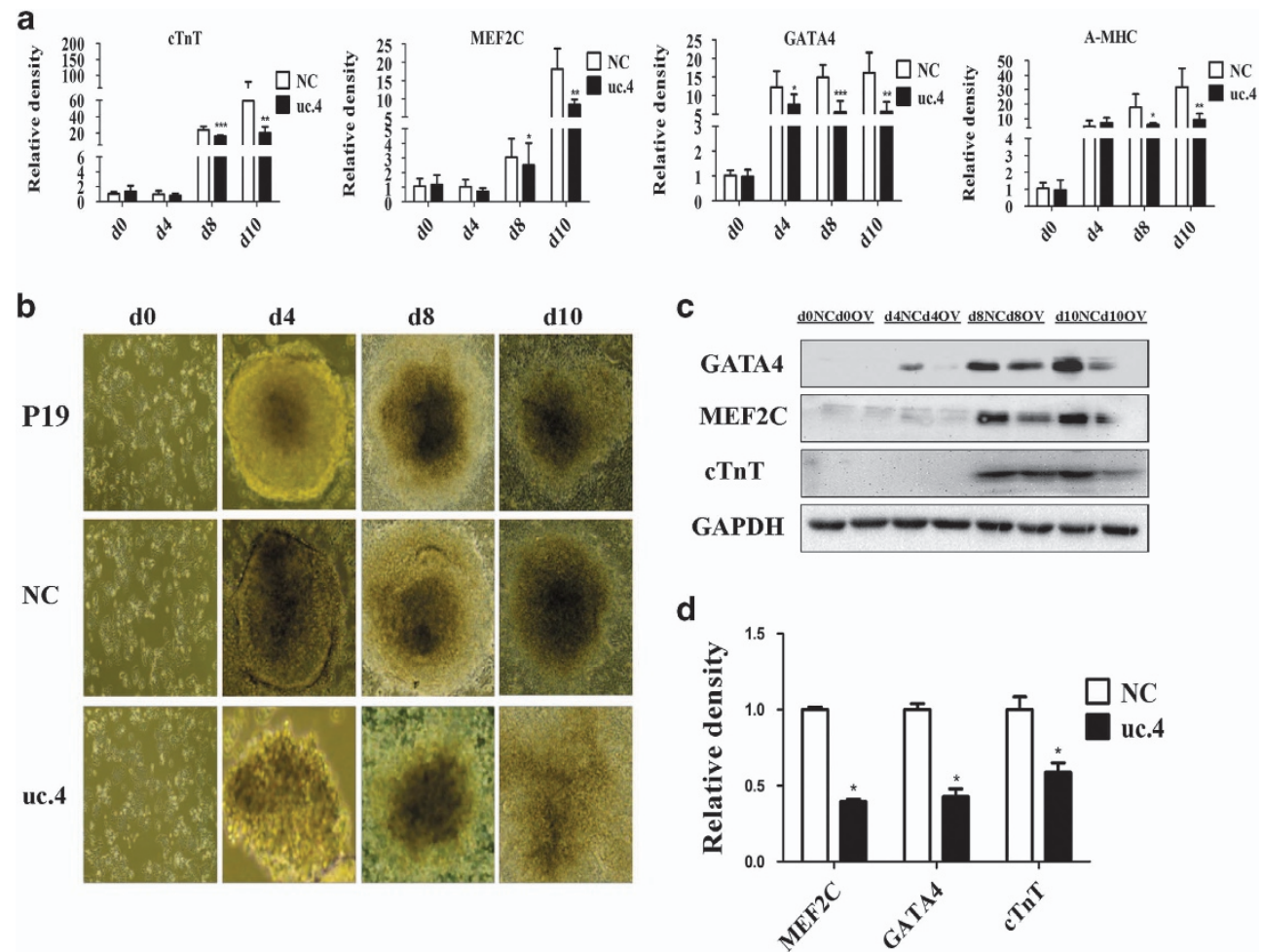

Figure 3 uc.4 overexpression influenced P19 cell differentiation. (a) qRT-PCR determination of the mRNA expression of cardiac markers (GATA4, cTnT, MEF2C, $\alpha-\mathrm{MHC}$ ) on days $0,4,8$ and 10 of differentiation. These differentiation-related genes were downregulated in the uc.4 overexpression group compared with the NC group. ${ }^{*} P<0.05,{ }^{*} P<0.01,{ }^{* *} P<0.001$ versus NC groups, Student's $t$-test. (b) Optical micrographs $(\times 100)$ of the differentiation process at $0,4,8$ and 10 days. (c) Western blot analysis of protein levels of cardiac markers (GATA4, cTnT and MEF2C) during differentiation on days 0, 4, 8, 10. OV: overexpression group. (d) The quantitative data are expressed as the relative intensity ratio of GATA4, cTnT and MEF2C to the expression of GAPDH on day $10 .{ }^{*} P<0.05$ versus NC groups, Student's $t$-test. Data are presented as the means \pm s.d. of three independent experiments. NC, negative control; qRT-PCR, quantitative real-time polymerase chain reaction.

the overexpression of uc.4 had a moderate effect on the overall morphological development of zebrafish embryos, which presented as developmental delays, heart looping defects and pericardial edema (Figure 4e). The relative position of the atrium and ventricle was slightly shifted. In subsequent observations, we found that the pericardium was gradually expanded and that the locomotion of the embryos and cardiac pump capacity were apparently weakened. The expression levels of the Homeobox protein Nkx2.5 (Nkx2.5), GATA4, Ventricular myosin heavy chain (Vmhc), Heart and neural crest derivative-expressed protein 2 (hand2), Mef2c and connexin43 (Cx43) were examined at $120 \mathrm{hpf}$ using qRT-PCR. A minor decrease was observed in these cardiac markers, suggesting that the overexpression of uc. 4 could negatively influence cardiac development (Figure 4f).

\section{Cardiac function and histological changes}

The heart rate was assessed at 48, 72 and $96 \mathrm{hpf}$ to evaluate the effects of uc.4 overexpression on cardiac contraction. The mean heart rates at $48 \mathrm{hpf}$ were $150.8 \pm 8.91$ beats per minute (bpm), $149.60 \pm 6.90$ and $111.73 \pm 3.92 \mathrm{bpm}$ for the WT, NC-injected and uc.4-injected embryos, respectively $(n=15)$. At $72 \mathrm{hpf}$, the mean heart rates were $179.33 \pm 9.79, \quad 177.20 \pm 9.65$ and
$159.60 \pm 7.53 \mathrm{bpm}$ for WT, NC-injected and uc.4-injected embryos, respectively $(n=15)$. At $96 \mathrm{hpf}$, the mean heart rates were $206.13 \pm 9.43,208.13 \pm 10.78$ and $179.73 \pm 13.41$ for the WT, NC-injected and uc.4-injected embryos, respectively $(n=15)$ (Figure 5a). These data indicated that the heart rate was markedly decreased in the uc.4 overexpression groups, which exhibited irregular heart contractions and weakened circulation. To further examine the cardiac phenotype, we analyzed the heart tissues using histological paraffin sections. Hearts from the control group showed an obvious twochamber structure, with a thicker ventricular wall than the atrial wall, and a relatively mature valve in the atrial-ventricular channel had formed. The overexpression group showed a thinner ventricular wall, and the valve structure was significantly smaller (Figure 5b). The distance between the sinus venous and the bulbus arteriosus (SV-BA) is often measured to assess the degree of cardiac cyclization. ${ }^{19}$ The SV-BA and edema index were increased in the overexpression group compared with the control group (Figures $5 \mathrm{c}$ and d). Taken together, these data demonstrated that the overexpression of uc.4 leads to evident changes in the function and structure of the embryonic heart. 
a

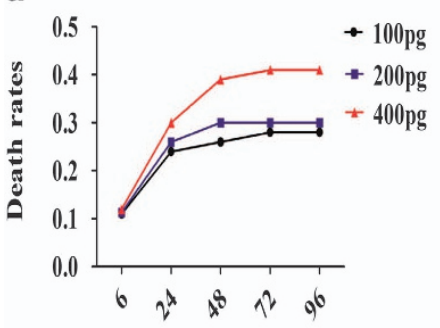

b

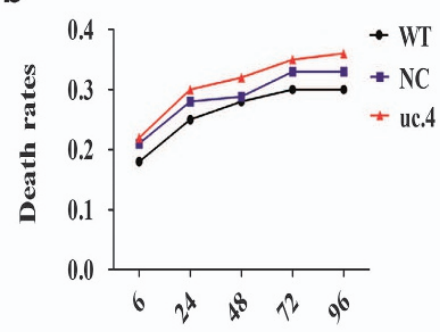

C

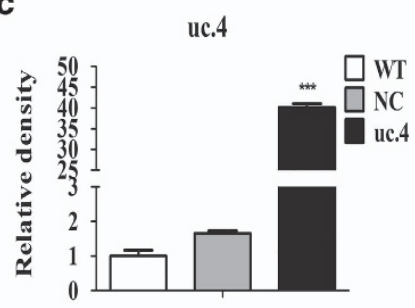

d

$400 X$

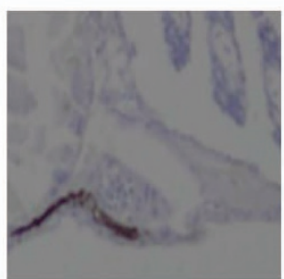

NC

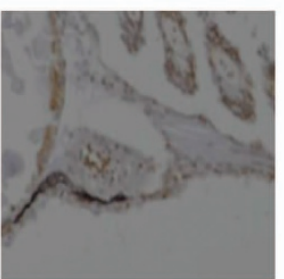

uc. 4

e

$48 h$
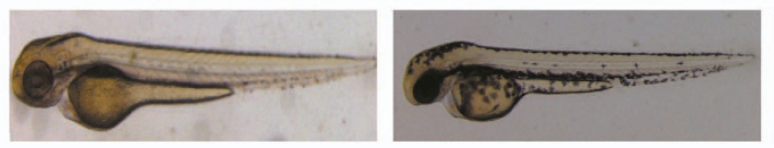

$72 \mathrm{~h}$
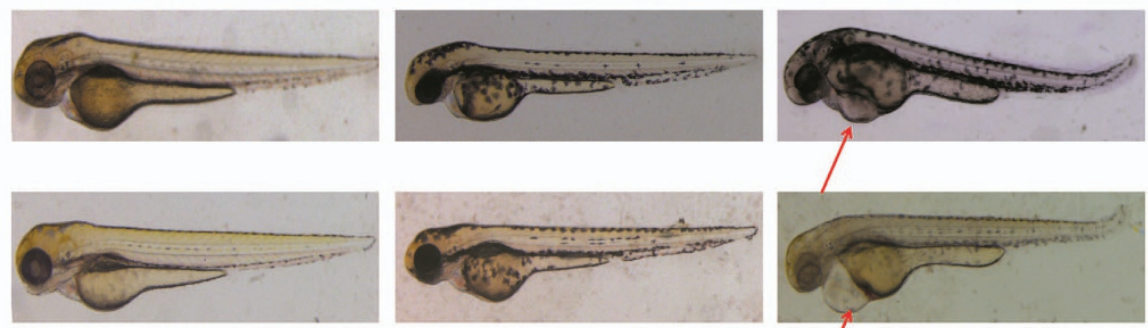

$96 \mathrm{~h}$

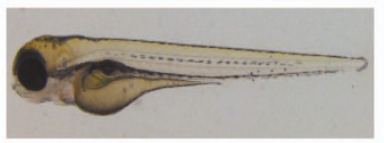

WT

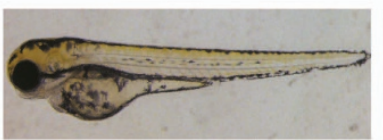

NC

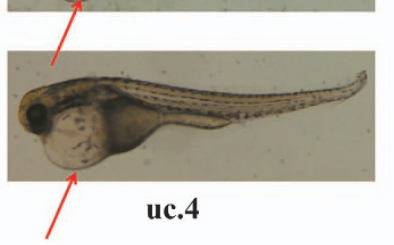

$\mathbf{f}$

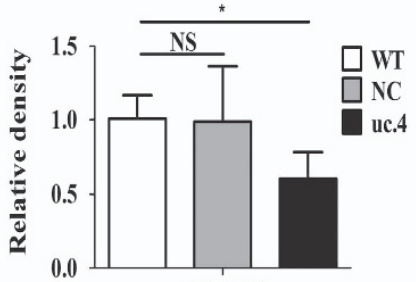

GATA4
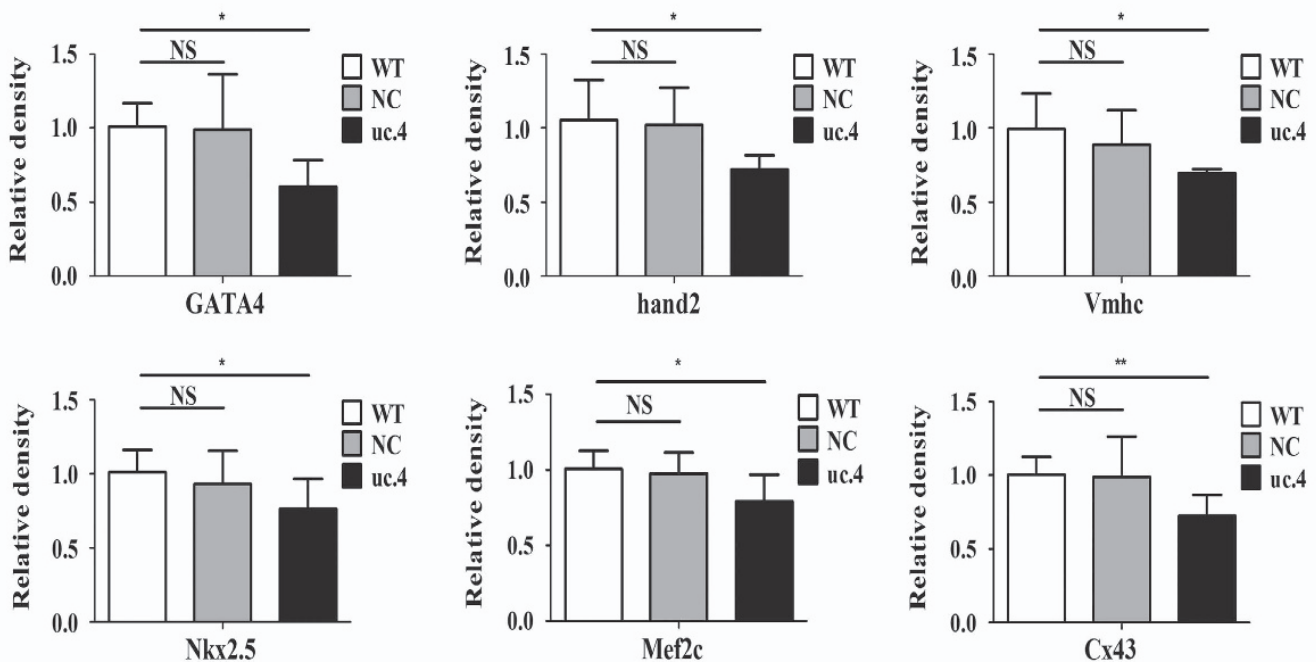

Figure 4 The overexpression of uc. 4 caused abnormal development of zebrafish. (a) The overexpression of uc. 4 increased the death rates of zebrafish in a dose-dependent manner. (b) The death rates of the WT, NC and uc.4 groups (200 pg). (c) The expression of uc.4 was upregulated in the uc.4 overexpression groups. ${ }^{* *} P<0.001$ versus NC groups, Student's $t$-test. (d) The overexpression of uc.4 was determined by in situ hybridization. uc.4 is primarily distributed in the atria of zebrafish. (e) The overexpression of uc.4 leads to heart looping defects and pericardial edema in zebrafish. (f) qRT-PCR determination of the mRNA expression of cardiac-specific markers (Nkx2.5, Vmhc, GATA4, hand2, Mef2c, Cx43). A minor decrease was observed in the expression of these cardiac markers. ${ }^{*} P<0.05$, ${ }^{*} P<0.01$ versus NC groups, Student's $t$-test. Each experiment was repeated three times. Data are presented as the means $\pm s . d$. NC, negative control; qRT-PCR, quantitative real-time polymerase chain reaction. 

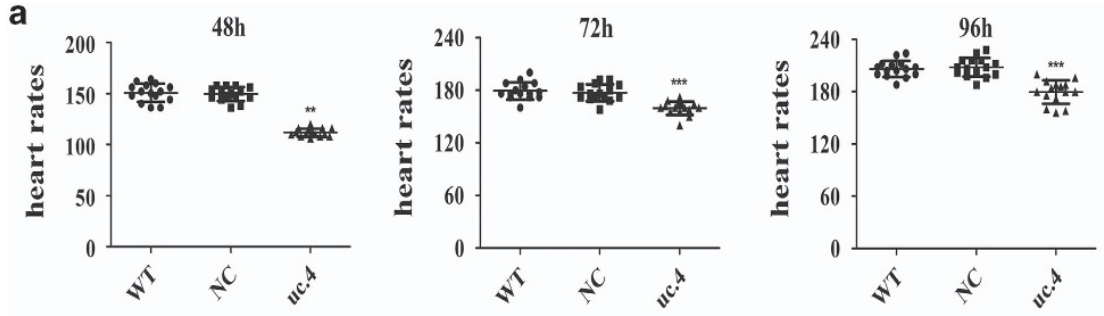

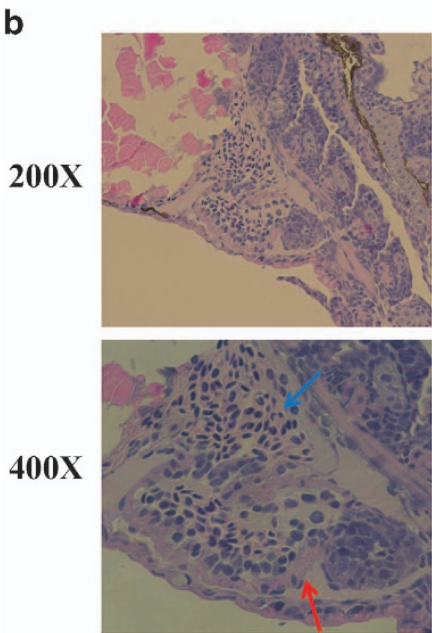

WT

C

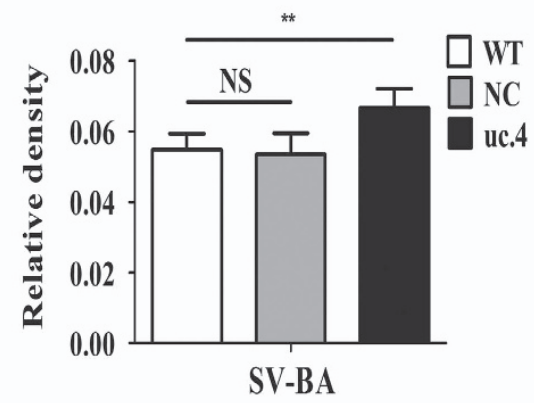

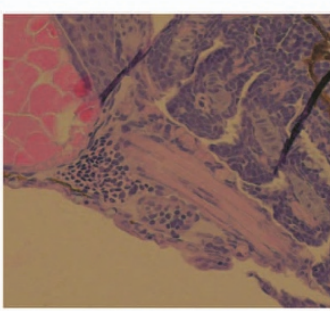

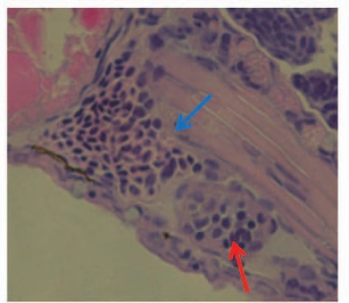

NC
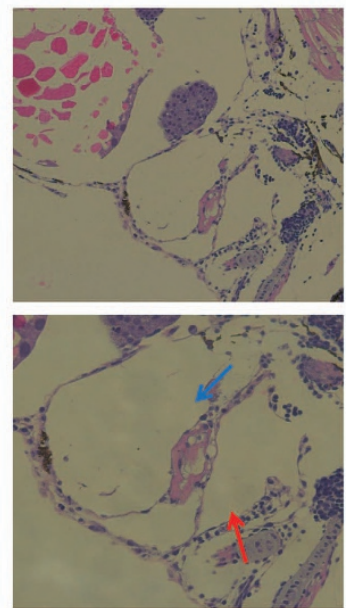

uc.4

d

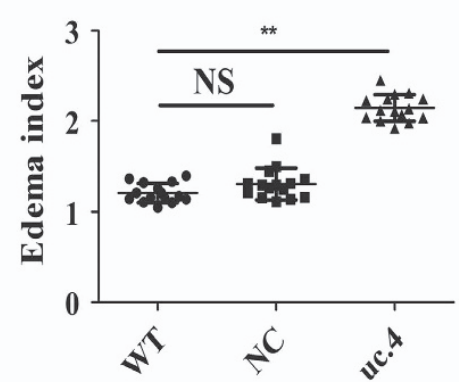

Figure 5 Cardiac function and histological changes. (a) The overexpression of uc.4 decreased the heart rates of zebrafish embryos. (b) Histological sections of the heart showed a thinner ventricular wall and smaller valve structure compared to control embryos. Red arrows: ventricle. Blue arrows: atrium. (c) SV-BA distance, which is normalized to total body length, resulting in a ratio. The SV-BA was increased in the overexpression group compared with the WT group. (d) The edema index was increased in the overexpression group compared with the WT group. ${ }^{* *} P<0.01,{ }^{* *} P<0.001$ versus WT groups, Student's $t$-test. Each experiment was repeated three times. Data are presented as the means \pm s.d. SV-BA, distance between the sinus venous and the bulbus arteriosus; WT, wild type.

uc.4 is expressed independent of the CASZ1 gene

According to the classification principle of lncRNAs, uc.4 is an intronic antisense $\operatorname{lncRNA} .{ }^{20}$ In humans, the uc. 4 transcript is situated within the third intron of the CASZ1 gene (Figure 6a), which maps to chromosome $1 \mathrm{p} 36.22$. Many researchers have found that CASZ1 is essential for cardiac morphogenesis and development. ${ }^{21}$ To assess the possible inter-relationship between CASZ1 and uc.4 transcription, we first examined the expression of CASZ1 by qRT-PCR during differentiation. The primers used to examine expression spanned a genomic region that included CASZ1 exon 5-6, which is $3.1 \mathrm{~kb}$ from uc.4 (Supplementary Figure 1C). CASZ1 expression was unchanged during differentiation (Figure 6b). These observations confirm that uc.4 is not expressed as part of the CASZ1 gene.

\section{Functional expression analysis of uc.4-regulated genes}

To further understand the mechanism by which uc. 4 alters the differentiation of P19 cells, we conducted a gene chip analysis of differentiated, uc.4-overexpressing cells (Figure 6c). We found that 1765 genes are differentially expressed $(P<0.05)$, including 1078 upregulated genes and 687 downregulated genes. All of these genes were associated with the negative regulation of muscle adaptation, His-Purkinje system development, coronary artery morphogenesis, cardiac ventricle 
a

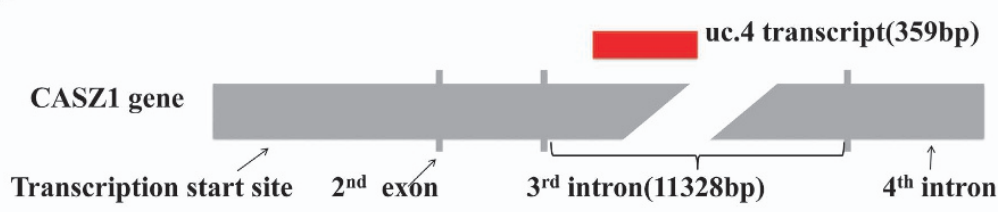

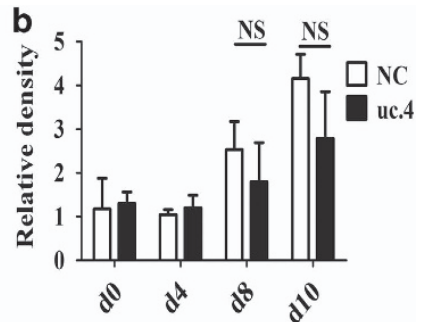

C

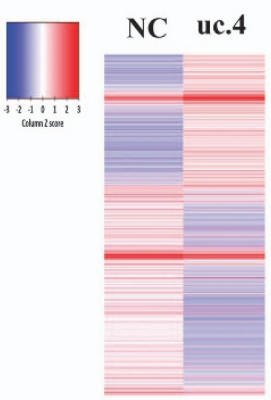

d
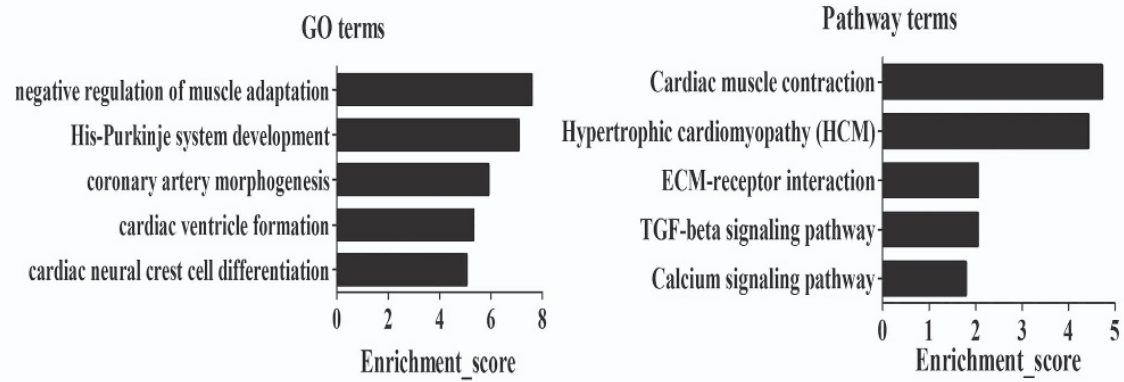
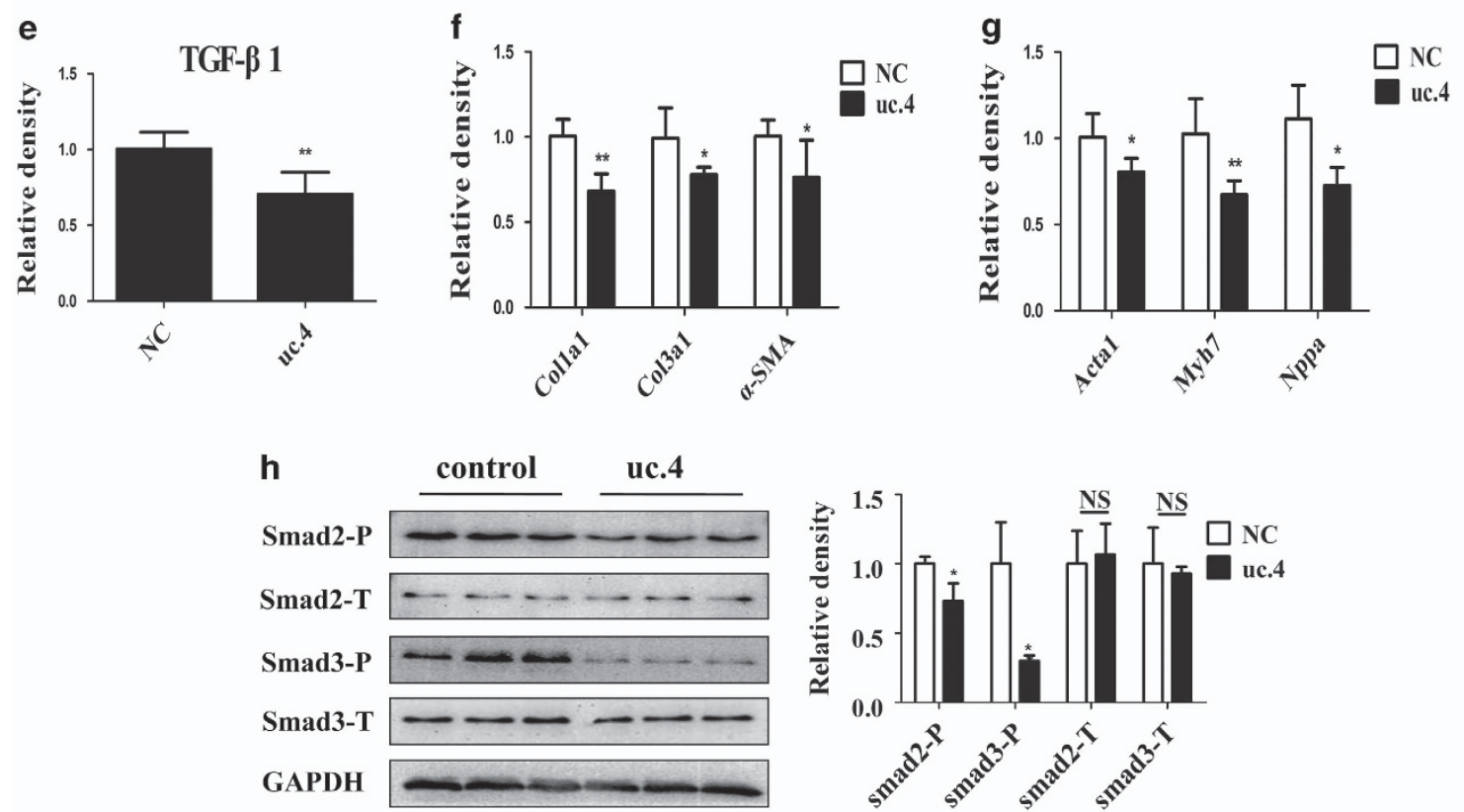

Figure 6 uc.4 regulates cell differentiation through the TGF-beta signaling pathway. (a) Schematic representation of the intronic localization of uc.4 within CASZ1. (b) uc.4 is expressed independently of the CASZ1 gene. NS, no significant difference. (c) Hierarchical clustering shows changes in the mRNA expression profile in the uc.4 overexpression groups. (d) The top enriched gene ontology (GO) biological processes of genes that show significant differences in mRNA expression upon uc.4 overexpression relative to controls. The top enriched pathway terms of genes that show significant differences in mRNA expression upon uc.4 overexpression relative to controls. (e) TGF-b1 levels were slightly decreased in the uc.4 overexpression group. (f) ECM-related genes showed decreased expression in the uc.4 overexpression group. (g) The Hypertrophic cardiomyopathy marker exhibited decreased expression in the uc.4 overexpression group. (h) uc.4 suppressed the phosphorylation of Smad2 and Smad3, without affecting total protein levels of Smad2/Smad3. The quantitative data are expressed as the relative intensity ratio of Smad2-P, Smad3-P, Smad2-T and Smad3-T to that of GAPDH. ${ }^{*} P<0.05,{ }^{* *} P<0.01$ versus NC groups, Student's $t$-test. The data are presented as the means \pm s.d. of three independent experiments. NC, negative control.

formation and cardiac neural crest cell differentiation, all of which are associated with heart development (Figure 6d). We also discovered that uc.4 is involved in cardiac muscle contraction, hypertrophic cardiomyopathy (HCM), ECMreceptor interactions, TGF-beta signaling and calcium signaling. A previous study suggested that TGF-b1 is closely associated with the extracellular matrix of the myocardium and is involved in both cell interstitial fibrosis and extracellular matrix deposition. ${ }^{22}$ The TGF-b1 levels were moderately decreased in the uc.4 overexpression group (Figure 6e). ECM-related genes and HCM markers were also decreased in the uc. 4 overexpression group (Figures $6 \mathrm{f}$ and g). The TGFbeta pathway has been shown to be critical for heart development, including specification of the cardiomyocyte lineage and 
the epithelial-mesenchymal transformation of the endocardium. ${ }^{23,24}$ We found that uc. 4 could modestly suppress Smad2 and Smad3 phosphorylation without affecting total protein levels of Smad2/Smad3 (Figure 6h).

\section{DISCUSSION}

In the present study, we identified a novel lncRNA uc.4 and verified its function in differentiation and heart development in zebrafish. In addition, we discovered that uc. 4 potentially regulates differentiation through the TGF-beta signaling pathway. The molecular network that mediates embryonic cardiac dysplasia is unclear; thus, additional efforts are required to identify the involved regulatory factors. Furthermore, exploring the role and mechanism of uc. 4 in cardiac development will ultimately open new avenues to understand heart development events during early embryogenesis.

The heart is the first organ to function during embryonic development. The complicated process of cardiac development demands highly specific spatiotemporal regulation of gene network activity. Thus, a better understanding of the pathways and specific factors involved in this process will be beneficial for manipulating these pathways for clinical treatments. Embryonic heart development involves the migration of precardiac stem cells, heart tube formation, cardiac looping, heart chamber formation and the development of the cardiac conduction system. The differentiation of cardiac cells primarily occurs when the heart tube transforms into a fourchambered functional human heart. ${ }^{25}$ The multipotent epicardial-derived cells differentiate into cardiac fibroblasts, endothelial cells and coronary smooth muscle cells depending on direction of transcription factor activation. ${ }^{26}$ Although thousands of lncRNAs have been characterized in various diseases, few have been functionally characterized, which has increased the scientific focus on their biological roles. Indeed, our data indicate that the overexpression of uc.4 and the consequent changes in gene expression are meaningful in embryonic cardiac development. Our results suggest that uc.4 suppresses the differentiation of P19 cells and that this lncRNA could function as a new class of molecular regulators within the gene network. Our work sheds new light onto how aberrant lncRNA expression correlates with disease. Therefore, we believe that understanding the exact role of lncRNAs in gene networks and diseases might provide new potential targets for the development of human therapeutics.

LncRNAs are known to regulate the expression of adjacent genes as cis-acting regulatory elements. However, we found no correlation between the expression of uc. 4 and its host gene CASZ1. Approximately 3\% of human lncRNAs are associated with their host or nearby genes. ${ }^{27}$ In this respect, we believe that uc. 4 does not act via CASZ1 to inhibit the P19 cell differentiation. It is tempting to speculate that the mechanism of uc. 4 may depend on its molecular localization. As most lncRNAs are localized to the nuclear compartment, the primary function of these molecules likely involves influencing transcription. ${ }^{28,29}$ The mechanisms by which this is achieved include bridging proteins and modifying chromatin. ${ }^{30}$
LncRNAs in the cytoplasmic function as molecular decoys, scaffolds or microRNA sponges at the post-transcriptional level. The TGF-beta signaling pathway is essential for a wide range of cellular responses, including proliferation, differentiation and apoptosis. The precise regulation of these cellular events is vital for normal heart development. ${ }^{31}$ Our findings show a role of uc.4 in cardiac development, and further experimentation on the potential mechanism of uc.4 and its effects on differentiation are clearly warranted.

In summary, we have elucidated the biological features and function of uc.4, which was expressed in abnormal embryonic heart tissues, and detailed its possible roles in embryonic heart development. Furthermore, uc. 4 regulates cell differentiation through the TGF-beta signaling pathway, a pivotal signaling pathway for cardiac development. LncRNAs have been shown to be associated with cancer development, ${ }^{32}$ demonstrating potential applications as novel diagnostic markers in clinical treatment, ${ }^{33}$ as well as direct targets for therapeutic intervention. ${ }^{34}$ Understanding the precise role of uc.4 during heart development might ultimately shed new light on therapeutics for CHD. Our data provide a first glimpse of a novel functional lncRNA and provide a better understanding of the molecular mechanisms of CHD.

\section{CONFLICT OF INTEREST}

The authors declare no conflict of interest.

\section{ACKNOWLEDGEMENTS}

This work was supported by the National Natural Science Foundation of China (81570209).

Author contributions: Yun Li and Lingmei Qian designed the research. Zi Jie Cheng, An Wen Yin and Hai Lang Liu performed the experiments. Hua Li and Meng Wen Feng analyzed the data. Zi Jie Cheng and Qi Jun Zhang wrote this manuscript. Lingmei Qian supervised this work. All of the authors read and approved the final manuscript.

\section{PUBLISHER'S NOTE}

Springer Nature remains neutral with regard to jurisdictional claims in published maps and institutional affiliations.

1 Gelb B, Brueckner M, Chung W, Goldmuntz E, Kaltman J, Kaski JP et al. The Congenital Heart Disease Genetic Network Study: rationale, design, and early results. Circ Res 2011; 112: 698-706.

2 Dolk H, Loane M, Garne E. Congenital heart defects in Europe: prevalence and perinatal mortality, 2000 to 2005. Circulation 2011; 123: 841-849.

3 Marino BS, Lipkin PH, Newburger JW, Peacock G, Gerdes M, Gaynor JW et al. Neurodevelopmental outcomes in children with congenital heart disease: evaluation and management: a scientific statement from the American Heart Association. Circulation 2012; 126: 1143-1172.

4 Derrien T, Johnson R, Bussotti G, Tanzer A, Djebali S, Tilgner H et al. The GENCODE v7 catalog of human long noncoding RNAs: analysis of their gene structure, evolution, and expression. Geno Res 2012; 22: 1775.

5 Fiedler J, Breckwoldt K, Remmele CW, Hartmann D, Dittrich M, Pfanne A et al. Development of long noncoding RNA-based strategies to modulate tissue vascularization. J Am Coll Cardiol 2015; 66: 2005-2015.

6 Zhang G, Wu Y, Xu D, Yan X, Long Noncoding RNA. ufc1 promotes proliferation of chondrocyte in osteoarthritis by acting as a sponge for miR-34a. DNA Cell Biol 2016; 35: 691-695. 
7 Reddy MA, Chen Z, Park JT, Wang M, Lanting L, Zhang Q et al. Regulation of inflammatory phenotype in macrophages by a diabetes-induced long noncoding RNA. Diabetes 2014; 63: 4249.

8 Kim T, Jeon YJ, Cui R, Lee JH, Peng Y, Kim SH et al. Role of MYCregulated long noncoding $\mathrm{RNAs}$ in cell cycle regulation and tumorigenesis. J Natl Cancer Inst 2015; 107: dju505.

9 Bejerano G, Pheasant M, Makunin I, Stephen S, Kent WJ, Mattick JS et al. Ultraconserved elements in the human genome. Science 2004; 304: 1321.

10 Cui X, You L, Li Y, Zhu L, Zhang F, Xie K et al. A transcribed ultraconserved noncoding RNA, uc.417, serves as a negative regulator of brown adipose tissue thermogenesis. FASEB J 2016; 30: 4301.

11 Carotenuto P, Fassan M, Pandolfo R, Lampis A, Vicentini C, Cascione L et al. Wnt signalling modulates transcribed-ultraconserved regions in hepatobiliary cancers. Gut 2016; 66: 312278.

12 Ferdin J, Nishida N, Wu X, Nicoloso MS, Shah MY, Devlin C et al. HINCUTs in cancer: hypoxia-induced noncoding ultraconserved transcripts. Cell Death Differ 2013; 20: 1675-1687.

13 Klattenhoff CA, Scheuermann JC, Surface LE, Bradley RK, Fields PA, Steinhauser $\mathrm{ML}$ et al. Braveheart, a long noncoding RNA required for cardiovascular lineage commitment. Cell 2013; 152: 570-583.

14 Grote P, Wittler L, Hendrix D, Koch F, Währisch S, Beisaw A et al. The tissue-specific IncRNA Fendrr is an essential regulator of heart and body wall development in the mouse. Dev Cell 2013; 24: 206-214.

15 Kurian L, Aguirre A, Sanchomartinez I, Benner C, Hishida T, Nguyen TB et al. Identification of novel long noncoding RNAs underlying vertebrate cardiovascular development. Circulation 2015; 131: 1278-1290.

16 Scheuermann JC, Boyer LA. Getting to the heart of the matter: Iong noncoding RNAs in cardiac development and disease. EMBO J 2013; 32 1805-1816.

17 Song G, Shen Y, Zhu J, Liu H, Liu M, Shen YQ et al. Integrated analysis of dysregulated IncRNA expression in fetal cardiac tissues with ventricular septal defect. PLOS ONE 2013; 8: e77492.

18 Kimmel CB, Ballard WW, Kimmel SR, Ullmann B, Schilling TF. Stages of embryonic development of the zebrafish. Dev Dyn 1995; 203: 253-310.

19 Antkiewicz DS, Burns CGCarney SA, Peterson RE, Heideman W. Heart malformation is an early response to TCDD in embryonic zebrafish. Toxicol Sci 2005; 84: 368

20 Ponting CP, Oliver PL, Reik W. Evolution and functions of long noncoding RNAs. Cell 2009; 136: 629.

21 Sojka S, Amin NM, Gibbs D, Christine KS, Charpentier MS, Conlon FL. Congenital heart disease protein 5 associates with CASZ1 to maintain myocardial tissue integrity. Development 2014; 141: 3040.

22 Fan D, Takawale A, Lee J, Kassiri Z. Cardiac fibroblasts, fibrosis and extracellular matrix remodeling in heart disease. Fibrogenesis Tissue Repair 2012; 5: 15 .

23 Sucov HM. molecular insights into cardiac development. Annu Rev Physiol 1998; 60: 287.
24 Blyszczuk $P$, Müller-Edenborn $B$, Valenta $T$, Osto $E$, Stellato $M$, Behnke $\mathrm{S}$ et al. Transforming growth factor- $\beta$-dependent Wnt secretion controls myofibroblast formation and myocardial fibrosis progression in experimental autoimmune myocarditis. Eur Heart J 2017; 38: 1413.

25 Birket MJ, Ribeiro MC, Verkerk AO, Ward D, Leitoguinho AR, den Hartogh SC et al. Expansion and patterning of cardiovascular progenitors derived from human pluripotent stem cells. Nat Biotechnol 2015; 33: 970.

26 Mitchell ME, Sander TL, Klinkner DB, Tomitamitchell A. The molecular basis of congenital heart disease. Semin Thorac Cardiovasc Surg 2007; 19: 228

27 Ulitsky I, Bartel DP. lincRNAs: genomics, evolution, and mechanisms. Cell 2013; 154: 26.

28 Reik W. Evolution and functions of long noncoding RNAs. Cell 2009; 136 : 629-641.

29 Rinn JL, Chang HY. Genome regulation by long noncoding RNAs. Annu Rev Biochem. 2012; 81: 145

30 Mercer TR, Dinger ME, Mattick JS. Long non-coding RNAs: insights into functions. Nat Rev Genet 2009; 10: 155-159.

31 Zhou Y, Cashman TJ, Nevis KR, Obregon P, Carney SA, Liu Y et al. Latent TGF $\beta$ binding protein 3 identifies a second heart field in zebrafish. Nature 2011; 474: 645.

32 Evans JR, Feng FY, Chinnaiyan AM. The bright side of dark matter: IncRNAs in cancer. J Clin Invest 2016; 126: 2775.

33 Chen G, Cao Y, Zhang L, Ma H, Shen C, Zhao J. Analysis of long non-coding RNA expression profiles identifies novel IncRNA biomarkers in the tumorigenesis and malignant progression of gliomas. Oncotarget 2017; 8: 67744.

34 Takahashi K, Yan I, Haga H, Patel T. Long noncoding RNA in liver diseases. Hepatology 2014; 60: 744-753.

This work is licensed under a Creative Commons Attribution-NonCommercial-ShareAlike 4.0 International License. The images or other third party material in this article are included in the article's Creative Commons license, unless indicated otherwise in the credit line; if the material is not included under the Creative Commons license, users will need to obtain permission from the license holder to reproduce the material. To view a copy of this license, visit http:// creativecommons.org/licenses/by-nc-sa/4.0/

C) The Author(s) 2018

Supplementary Information accompanies the paper on Experimental \& Molecular Medicine website (http://www.nature.com/emm) 